\title{
XXIX. Notes on the absorption and scattering of X- rays, and the characteristic radiations of J series
}

\section{C.G. Barkla F.R.S. \& Margaret P. White M.A. B.Sc.}

To cite this article: C.G. Barkla F.R.S. \& Margaret P. White M.A. B.Sc. (1917) XXIX. Notes on the absorption and scattering of X-rays, and the characteristic radiations of J series, Philosophical Magazine Series 6, 34:202, 270-285, DOI: 10.1080/14786441008635704

To link to this article: http://dx.doi.org/10.1080/14786441008635704

曲 Published online: 08 Apr 2009.

Submit your article to this journal $\lceil\pi$

Џ Article views: 13

Q View related articles $\square$

Citing articles: 13 View citing articles 5 
requires more careful installation for the best work in the laboratory. It consists of a simple pendulum, with a light lath for its rod, from a movable stud on which hangs the cord pendulum.

Even if the lengths are made equal, and also the masses, the vibrations of this pendulum are not interchangeable. The effects of various initial conditions are accordingly more striking.

It may be used for couplings varying from about 10 to 55 per cent.

Photographic reproductions are given of ten single and four double traces obtained with this pendulum.

4. The mathematical theories of both pendulums are developed and compared with each other and with the theory of the electrical case it is sought to represent. The experiments are in satisfactory agreement with theory, though this is not always immediately obvious.

5 . In the experiments and most of the theory the lengths of the pendulums have been equal and also the masses of the bobs. A large field lies ready for exploration in the cases where the quantities of both classes are unequal and varied at will. These cases are reserved for later papers.

University College, Nottingham, July 18, 1917.

XXIX. Notes on the Absorption and Scattering of $X$-Rays, and the Characteristic Radiations of $J$ Series. By C. G. Barkla, F.R.S., and Margaret P. White, M.A., B.Sc., University of Edinburgh*.

\section{Introduction.}

$7 \mathrm{HE}$ experimental investigation of the absorption of X-rays of short wave-length is of importance in a study both of the theories of electromagnetic radiation and of atomic structure. The experimental work on this subject, the results of which are given in this paper, was suggested-indeed forced upon us-by the discovery that the observed intensity of corpuscular radiation from air was not what was to be expected on the simple theory of emission of fluorescent and corpuscular radiations as given in the Bakerian Lecture for $1916 \dagger$. An irregularity appeared in the results which could be explained on the assumption that the light elements emitted under suitable stimulus a hitherto unobserved characteristic X-radiation-(a J radiation). Such

* Communicated by the Authors.

† Proc. Roy. Soc. A. xcii. May 25, 1916 ; Phil. Trans. A. 217. pp. $315-$ 360. Preliminary notes in 'Nature,' Feb. 18 and March 4, 1915. 
a radiation was looked for and found, as already briefly announced. The evidence obtained from absorption experiments is given below*.

But in addition, there are in these results a number of other features which are of considerable theoretical interest, and to which attention should be drawn.

\section{Experiments.}

Of the experiments little need be said. The precautions essential to the accurate determination of an absorption coefficient have frequently been stated $\dagger$. The principal danger is that of allowing secondary rays of any type from the absorbing substance to enter the ionization chamber in which the intensity of transmitted radiation is measured.

The absorption coefficient $\mu$ of a homogeneous radiation is defined by the equation $I=I_{0} e^{-\mu x}$, where $I$ is the intensity of the primary radiation of initial intensity $I_{0}$ still proceeding as primary radiation after traversing a thickness $x$ of absorbing materialf.

In these experiments the radiation employed was almost invariably a primary radiation from which the more easily absorbed constituents had been previously eliminated. In the case of the most penetrating radiations, the remaining radiation upon which the experiments were made was remarkably homogeneous. In order to reduce any error due to heterogeneity to a minimum, the thickness of the absorbing sheet was adjusted to absorb approximately the same fraction (50 per cent.) of the incident radiation.

A few experiments were also made with characteristic radiations ( $\mathrm{K}$ series) as primary radiations. The results obtained agreed almost perfectly with those obtained with the primary radiations direct from an X-ray tube $\S$.

* Indications of the existence of such a radiation had been obtained by one of us many years before. Some of these were recorded; others not. But the evidence was insufficient to show either that the radiation emitted was really a characteristic radiation or that it could be in any way identified or classified. Indeed the probable significance of these results had been lost sight of until associated irregularities became a serious hindrance to the development of the theory. It seems highly probable that these $J$ radiations account in part for some of the discrepancies between the results obtained by some experimenters and those of the writer on the scattered radiation. By adjustment of the primary radiation care had been taken by the writer to keep the scattered radiation free from admixture with such a characteristic radiation.

† Barkla and Sadler, Phil. Mag. May 1909.

$\pm \mu$ is a measure of the rate of loss of intensity of the transmitted beam, whether by scattering or any other process.

\$ Except at a "discontinuity" where absorptions are exceedingly sensitive to small variations of wave-length. In this case the discrepancy was a regular one. 
As absorbing substances, copper, aluminium, water, paraffinwax, and filter-paper were used. The results are given in Table I.

TABLE I.

\begin{tabular}{|c|c|c|c|c|c|c|}
\hline $\begin{array}{c}\text { Wave-length } \\
\text { (Hull \& Rice). }\end{array}$ & $\begin{array}{l}\text { Wave-length } \\
\text { (Siegbahn). }\end{array}$ & $\begin{array}{c}(\mu / \rho) \\
\text { Copper. }\end{array}$ & $\begin{array}{c}(\mu / \rho) \\
\text { Aluminium. }\end{array}$ & $\begin{array}{l}(\mu / \rho) \\
\text { Water. }\end{array}$ & $\begin{array}{c}(\mu / \rho) \\
\text { Paper. }\end{array}$ & $\begin{array}{c}(\mu / \rho)^{\prime} \\
\text { Paraffin-W ax. }\end{array}$ \\
\hline 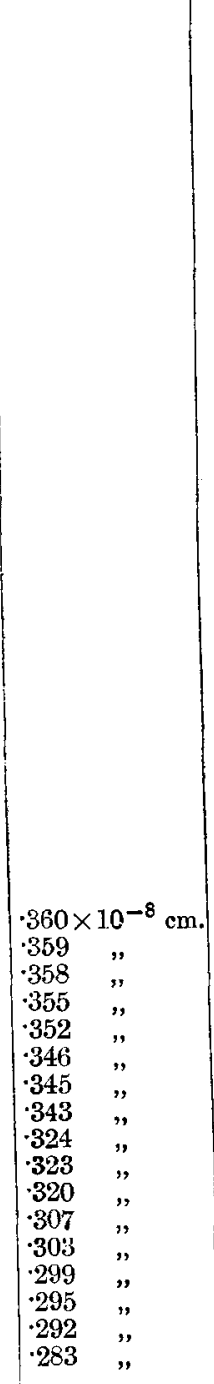 & 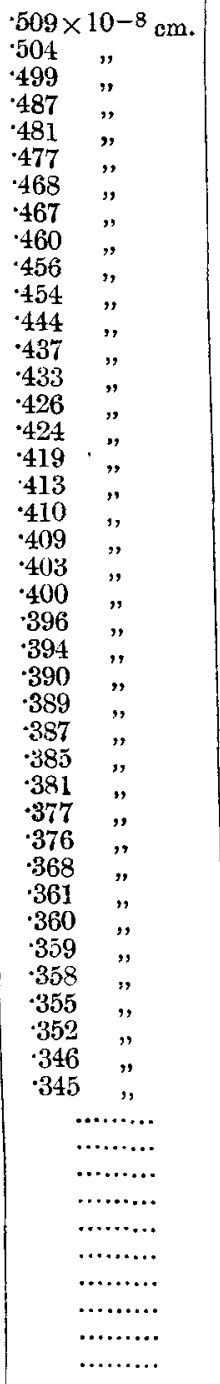 & $\begin{array}{c}19 \cdot 05 \\
18 \cdot 10 \\
17 \cdot 7 \\
16 \cdot 4 \\
15 \cdot 8 \\
15 \cdot 4 \\
14 \cdot 55 \\
14 \cdot 40 \\
13 \cdot 75 \\
13 \cdot 25 \\
13 \cdot 10 \\
12 \cdot 20 \\
11 \cdot 6 \\
11 \cdot 2 \\
10 \cdot 55 \\
10 \cdot 40 \\
9 \cdot 98 \\
9 \cdot 60 \\
9 \cdot 31 \\
9 \cdot 28 \\
8 \cdot 76 \\
8 \cdot 55 \\
8 \cdot 24 \\
8 \cdot 04 \\
7 \cdot 82 \\
7 \cdot 77 \\
7 \cdot 61 \\
7 \cdot 47 \\
7 \cdot 15 \\
6 \cdot 94 \\
6 \cdot 90 \\
6 \cdot 38 \\
5 \cdot 98 \\
5 \cdot 92 \\
5 \cdot 87 \\
5 \cdot 81 \\
5 \cdot 58 \\
5 \cdot 40 \\
5 \cdot 17 \\
5 \cdot 00 \\
4 \cdot 92 \\
4 \cdot 71 \\
4 \cdot 70 \\
4 \cdot 54 \\
4 \cdot 02 \\
3 \cdot 77 \\
3 \cdot 71 \\
3 \cdot 68 \\
3 \cdot 47 \\
3 \cdot \cdot 0\end{array}$ & 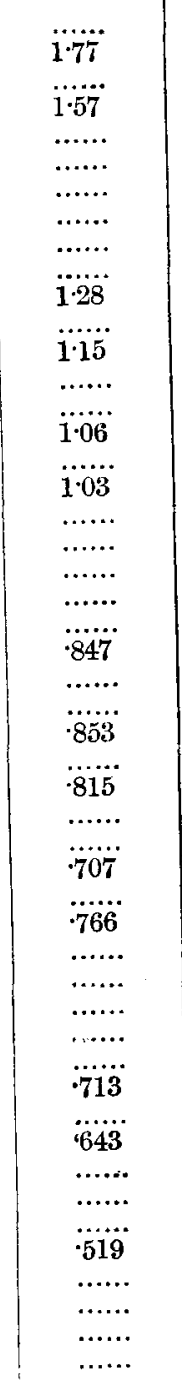 & 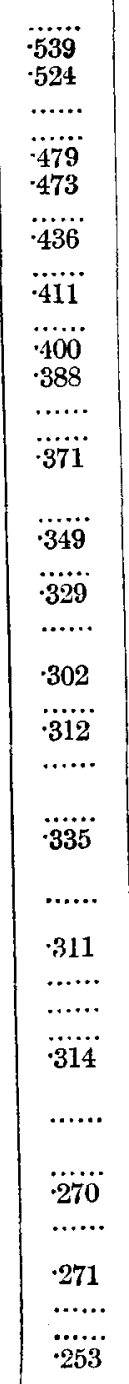 & $\begin{array}{l}\ldots \ldots \\
.353 \\
\ldots \ldots \\
\\
.328 \\
\ldots \ldots \\
.389 \\
\ldots \ldots \\
\\
\cdot 361 \\
\ldots . . . \\
\ldots \ldots \\
.330 \\
\ldots \ldots . \\
\ldots \ldots . \\
.309\end{array}$ & $\begin{array}{l}\cdot 279 \\
.267 \\
\cdot 263 \\
\cdot 252 \\
\cdot 266\end{array}$ \\
\hline
\end{tabular}




\begin{tabular}{|c|c|c|c|c|c|c|}
\hline $\begin{array}{l}\text { Wave-length } \\
\text { (Hull \& Rice). }\end{array}$ & $\begin{array}{l}\text { Wave-leugth } \\
\text { (Siegbahn) }\end{array}$ & $\begin{array}{c}(\mu / \rho) \\
\text { Copper. }\end{array}$ & $\begin{array}{c}(\mu / \rho) \\
\text { Aluminium }\end{array}$ & $\begin{array}{l}(\mu / \rho) \\
\text { Water. }\end{array}$ & $\begin{array}{c}(\mu / \rho) \\
\text { Paper. }\end{array}$ & $\begin{array}{c}(\mu / \rho) \\
\text { Paraffin-Wax }\end{array}$ \\
\hline 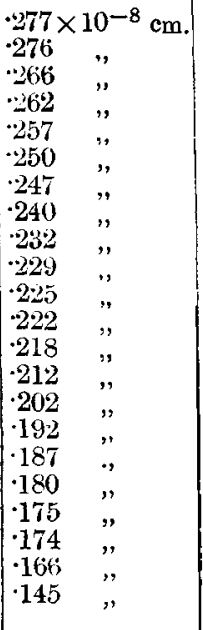 & 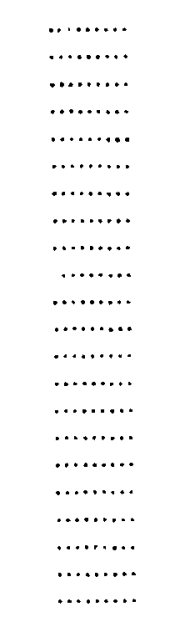 & $\begin{array}{l}3 \cdot 08 \\
3 \cdot 06 \\
2 \cdot 77 \\
2 \cdot 62 \\
2 \cdot 58 \\
2 \cdot 33 \\
2 \cdot 27 \\
2 \cdot 10 \\
1 \cdot 94 \\
1 \cdot 86 \\
1 \cdot 77 \\
1 \cdot 71 \\
1 \cdot 66 \\
1 \cdot 54 \\
1 \cdot 40 \\
1 \cdot 29 \\
1 \cdot 20 \\
1 \cdot 10 \\
1 \cdot 03 \\
1 \cdot 02 \\
.950 \\
\cdot 707\end{array}$ & 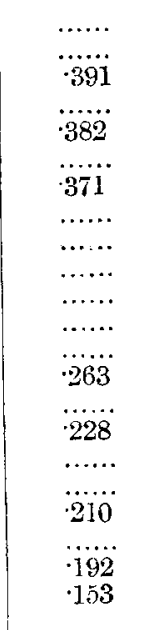 & 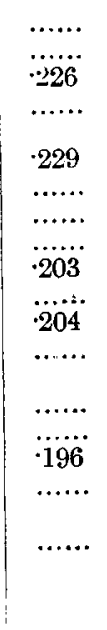 & $\begin{array}{l}\ldots \ldots * \\
\cdot 245 \\
\cdot 252 \\
\cdot 214 \\
\ldots \ldots \\
.227 \\
\ldots \ldots \ldots \\
\ldots \ldots \\
\ldots \ldots \\
.186\end{array}$ & $\begin{array}{r}\cdot 195 \\
\cdot 192\end{array}$ \\
\hline
\end{tabular}

For various reasons the absorbability of X-rays has usually been measured in aluminium. Apart from practical reasons, aluminium was one of the most suitable substances to use because no characteristic radiation from aluminium had been observed within the range of wave-lengths of X-rays in common use. In other words, the wave-length was a simple single-valued function of the absorption in aluminium. Our experiments, however, have shown that aluminium emits a characteristic radiation of wave-length about $\cdot 37 \times 10^{-8} \mathrm{~cm}$.; it is therefore not a suitable substance in which to measure the general absorbability of radiation through a range of wave-lengths such as that used in these experiments. The same objection does not hold in the case of copper, because its $\mathbf{K}$ spectral lines are fairly distant on the longer wave-length side and its I spectral lines (assuming they exist) must be somewhere on the shorter wave-length side of the $J$ radiations of the other substances dealt with.

We have, therefore, plotted absorptions in aluminium, water, filter-paper, and paraffin-wax as ordinates against absorptions in copper as abscissæ-thus taking the last-named as the standard of general absorbability.

The results are given in fig. 1 . The scale of ordinates for filter-paper has been made 6/5 times as great as for the other three. This was necessary as the water and paper 
absorptions were too nearly alike to show clearly on the same scale. The interesting features of these curves are described below.

Fig. 1.

Showing absorption curves in the neighbourhood of $\mathbf{J}$ spectral lines.

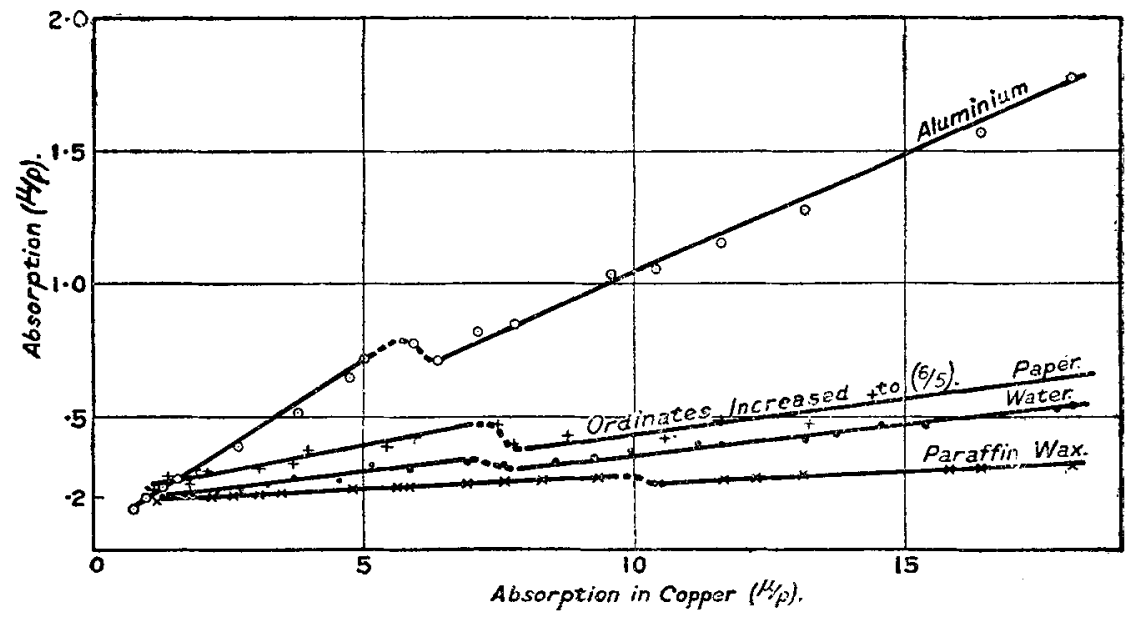

Characteristic Radiations of J Series.

The simplicity of the form of each curve is broken by a feature which indicates the existence of an X-radiation characteristic of each substance. Similar curves were first shown by Barkla and Sadler* in association with the $\mathrm{K}$-radiations, and by Barkla and Collier $\dagger$ in association with the L-radiations. No such irregularity has ever been observed except in association with a characteristic X-radiation.

The three "discontinuities," as we shall call them, being due to the elements aluminium, oxygen (in both water and paper), and carbon (in paraffin-wax), we see that the higher the atomic weight the more penetrating is the radiation at which the discontinuity occurs; in other words, the bigher the atomic weight of the element the higher is the frequency of its characteristic radiation. This is in harmony with the results obtained for $K$ and $L$ radiations.

[Any discontinuity in the absorption curve for hydrogen would be inappreciable and could not be detected in the curves for paper, water, and paraffin-wax, of which it is a constituent. That due to carbon is clearly marked in the 
curve for paraffin-wax; it must also occur in the curve for paper, but the "step" is only about half as big as in paraffinwax, and the fractional change is smaller still. There appears to be indication of this step (see fig. 1), but it is within the limits of experimental error in this case, and has therefore not been shown in the curve. It would indeed be barely appreciable in the figure.]

The wave-lengths obtained from the known relation between wave-length and absorption in aluminium are approximately*:

$$
\begin{aligned}
& \text { C } \ldots \ldots \cdot \cdot 42 \times 10^{-8} \mathrm{~cm} . \\
& \mathrm{O} \ldots \ldots \cdot 39 \quad, \\
& \text { Al..... }
\end{aligned}
$$

\section{Absorption, Scattering, Fluorescence.}

One of us has previously pointed out that an X-radiation loses energy by two independent processes. One is the process which we may briefly call scattering; the other is that which is associated with the emission of corpuscular (electronic) radiations and their accompanying fluorescent (characteristic) X-radiations.

The former absorption varies slowly with the wave-length -over certain regions it is approximately independent of wave-length; the latter varies very rapidly-over certain ranges it is approximately as the (wave-length) ${ }^{3}$.

The former-produced by equal masses of different elements-varies little with the atomic weight of the absorbing element; the latter considerably.

* These values are somewhat lower than those previously given. There is, however, some uncertainty as to the absolute values,--though not so much as to relative values--owing to the fact that reither the characteristic radiations nor the primary radiations used in these experiments were homogeneous.

The values previously given as $56 \times 10^{-8} \mathrm{~cm}$. for nitrogen and $5 \times 10^{-8}$ for sulphur were for the constituents of longest wave-length $\left(\alpha_{1}\right.$ lines), on the assumption that $\mathbf{J}$ radiations were of constitution similar to the $K$ radiations. There are indications that the $J$ radiations are more homogeneous than $\mathrm{K}$ radiations, and that the wave-lengths of the corresponding $\beta$ lines which are approximately $5 \times 10^{-8}$ and $44 \times 10^{-8} \mathrm{~cm}$. respectively, are more correctly the values to be assigned to nitrogen and sulphur by the ionization method.

There is thus a small discrepancy between the values obtained by the ionization and absorption methods, but the variation of $\lambda_{J}$ with atomic weight is about the same in both. In the ionization experiments $K$ characteristic radiations were used; in the absorption experiments, primary radiations. Equal average penetrating powers in these two radiations do not correspond to exactly the same effective wave-lengths. Greater accuracy will be obtained by the use of more homogeneous radiations, or by the interference method. 
As a consequence when the wave-length is small, scattering is the predominant cause of loss of energy except in the heaviest elements. In general, the lower the atomic weight the greater is the proportion of loss by scattering to the total loss of energy.

\section{Scattering and Absorption.}

In paraffin-wax, water, and paper scattering accounts for almost the whole "absorption" within the range of the shortest wave-lengths here dealt with. This is shown by the following facts :-

(1) The total absorption coefficient in each substance varies little with the wave-length of the radiation absorbed; (2) the total absorption coefficient is almost independent of the nature of the light-absorbing substance; (3) there is close agreement between the magnitude of the coefficient and that directly calculated from measurements of the energy of radiation scattered.

The small variation of absorption in light elements with a change in wave-length is illustrated by a comparison of the absorptions in paraffin-wax and in copper-the former increasing only by about 20 per cent., while the absorption in copper increases by 400 per cent.

The absorbability in light substances as paraffin-wax, paper, water, or even aluminium is thus very unsuitable as a measure of the character (wave-length) of such lighfrequency radiations even though the effect of scattering is not lost sight of, as it unfortunately has been both in the derivation and in the application of formulæ connecting wave-lengths and absorptions.

We see, too, that the absorption coefficients $(\mu \mid \rho)$ in paraffinwax, paper, and water all become only slightly less than $\cdot 2$ for X-radiation of very short wave-length. This agrees exceedingly well with the value $\cdot 2$ obtained many years ago* from measurement of the energy of the X-radiation of longer wave-length scattered in light substances (with the exception of hydrogen). Owing to the constituent hydrogen the scattering coefficients for these three substances as then determined for somewhat longer waves are slightly greater than $\cdot 2$.

Further, the value $\cdot 2$ agrees almost perfectly with that obtained by calculation, on the assumption that the number of electrons per atom is equal to the atomic number. Or, putting it another way, the value $\cdot 2$ when applied to Sir J. J. Thomson's calculated expression for the energy

* Barkla, Phil. Mag. May 1904. 
lost by scattering, gives with remarkable precision the number of electrons per atom now accepted. This method was in fact the first to lead to the present conclusion*. All these results verify not only the general laws of scattering which one of us arrived at many years ago, but they indicate also the accuracy of the energy measurement made at that time.

It will, however, be noticed that the coefficient of absorption in aluminium sinks quite appreciably below $\cdot 2$ to a value $\cdot 15$, and that there is a tendency to lower values still. Equally low values have been obtained by Rutherford, Richardson, and Barnes $\dagger$, and by Hull and Rice $\ddagger$ in experiments with the Coolidge tube.

In addition the values of the coefficients of absorption of $\gamma$ rays obtained by Soddy and Russell, Ishino $\S$, and others indicate that the simple law of scattering (approximate independence of wave-length) must break down for very short waves, for values of $\mu / \rho$ as low as $\cdot 04$ have been obtained.

We have already shown $\|$ that it does not hold for much longer waves, and that this is to be expected theoretically; that although in light elements the scattering varies little with wave-length, yet there is a slight increase with an increase in wave-length of the radiation; that this increase becomes decidedly marked when the wave-length becomes comparable with the size of an atom; that in heavy atoms the variation is most pronounced, the scattering increasing many fold; but that with shorter waves the mass scattering even in the heavier elements approaches equality with that in the light.

In view of the facts stated above, it certainly looks as though we have in the absorption by aluminium, evidence of the beginning of a second marked deviation from the simple laws-that is, evidence that a diminution of scattering and absorption is setting in when the wave-length becomes small. This appears to be the link connecting the absorption of $\mathrm{X}$-rays with the absorption of $\gamma$ rays. In this connexion it may be observed that the absorption of X-rays in aluminium appears to cross that in paraffin, paper, water, \&c., and to reach a lower value. This we should expect it to do as the

* As the values of $e / m$ and $e$ were obtained more accurately, redeterminations of the number of electrons per atom were made. See Barkla, Phil. Mag. May 1904; Jahrbuch der Radioalktivität, und Elektronik, A pril 1908; Phil. Mag. May 1911.

$\dagger$ Phil. Mag. Sept. 1915.

I. Phys. Review, Sept. 1916.

$\S$ Phil. Mag. Jan. 1917.

If Barkla and Dunlop, Phil. Mag. March 1916. 
other three substances through their constituent hydrogen contain slightly more electrons per unit mass, and so scatter to a greater extent mass for mass. Such a lower value apparently persists thronghout the results of all experiments on the absorption of $y$ rays (see Table II.).

TABLE II.

Coefficients of Absorption of $\gamma$ Rays. (Soddy \& Russell.)

\begin{tabular}{|c|c|c|c|c|}
\hline Absorbing Sheet. & $\begin{array}{c}\text { Thorium } \mathrm{D} \\
\boldsymbol{\mu} / \rho\end{array}$ & $\underset{\mu / \rho .}{\text { Radium }} \mathrm{C}$ & $\begin{array}{c}\text { Mesothorium } 2 . \\
\mu / \rho \text {. }\end{array}$ & $\underset{\mu / \rho .}{\text { Uranium }} \mathrm{X}$. \\
\hline 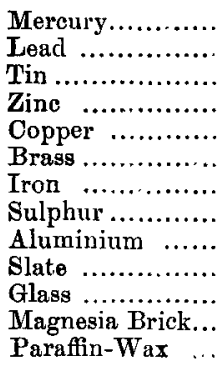 & $\begin{array}{l}\cdot 0405 \\
.0326 \\
\cdot 033 \\
\cdot 0334 \\
\cdot 0325 \\
\cdot 0328 \\
.0369 \\
\cdot 0324 \\
\cdot 0337 \\
\cdot 0352 \\
\cdot 0323 \\
\cdot 0361\end{array}$ & $\begin{array}{l}.0472 \\
.0438 \\
.0388 \\
.0393 \\
.0398 \\
.0389 \\
.0399 \\
.0438 \\
.0406 \\
.0414 \\
.0416 \\
\ldots \ldots . \\
.0464\end{array}$ & $\begin{array}{l}.0544 \\
.0421 \\
\cdot 0424 \\
.0423 \\
.0425 \\
.0415 \\
.0465 \\
.0421 \\
\ldots \ldots \\
.0448 \\
.0469 \\
.0580\end{array}$ & $\begin{array}{l}.0612 \\
.0636 \\
.0470 \\
.0465 \\
.0472 \\
.0470 \\
.0472 \\
.0316 \\
.0469 \\
.0469 \\
.0484 \\
.0478 \\
.0502\end{array}$ \\
\hline
\end{tabular}

Absorption and Scattering of $\gamma$ Rays.

The explanation of the approximate equality of the mass coefficients of absorption in different substances of X-rays of short wave-length thus leads to the explanation also of the approximate equality in various substances of the mass coefficient of absorption of $\gamma$ rays. The results with these short waves bridge the gap between the very different absorptions in various substances of $X$-rays of longer wavelength and the approximately equal absorptions of $\gamma$ rays in these substances. Evidently the absorption of $\gamma$ rays in substances of low atomic weight is almost entirely absorption by scattering, which is proportional to the number of electrons in the substance traversed *. Thus, as we seo from Table II., $\mu / \rho$ varies little with the atomic weight of the absorbing substance except in the case of the heaviest elements, in which the value may be as much as 40 per cent. greater.

It is prohable, however, that the "fluorescence absorption"

* Mr. Ishino thinks there is a large true absorption. This is probably true of mercury, the substance upon which he made further experiments. With regard to the lighter elements, all evidence appears to point the other way. 
is not negligible in these heavier substances, as it is not even in copper when high-frequency $X$-rays are employed (see Table I.).

A point worthy of notice is that as the wave-length of the $\mathrm{X}$-radiation diminishes, after the mass absorption coefficients in various substances become approximately equal-owing to the "fluorescence absorption" becoming very small- the absorptions diminish in different substances at approximately the same rate. This indicates that the scattering diminishes at approximately the same rate in different substances with a diminution of wave-length.

On the other hand, with longer waves the scattering increases with the wave-length at very different rates in different substances. This latter variation, as we have explained, is probably due to the electrons acting in groups rather than individually.

The variations observed-(or rather inferred from absorption experiments, for it has not been observed directly) -when the waves are short, must have another explanation. It may be connected with the fact that when the wave-length is short, the deviation of the observed distribution of X-rars scattered around the substance traversed from that given by the simple theory becomes very marked. It seems certain that scattering loses its simple character. The scattering of $\gamma$ and X-rays may thus be considered in three stages. With very short waves ( $\gamma$ rays) the scattering increases with the wave-length in all substances at approximately the same rate, until the mass scattering coefficient approaches $\cdot 2$.

Secondly, there is little or no variation of scattering with wave-length over a long range of wave-lengths in light elements, over a shorter range of wave-lengths in heavier elements.

Finally, the scattering increases with wave-length, slightly in light elements, more rapidly in heavier elements.

The second and third stages have already been explained. The first stage needs further investigation.

\section{Alsorption associated with $X$-ray Fluorescence.}

Absorption associated with the phenomenon of $\mathrm{X}$-ray fluorescence may be analysed into several distinct and evidently independent absorptions connected with various sets of electrons within the atom. Thus there is the $\mathbf{K}$ absorption which is that associated with the emission of a particular group of electrons which we call the $\mathrm{K}$ electrons because their emission is associated also with the emission 
of the $\mathrm{K}$ fluorescent $\mathrm{X}$-radiation ; similarly there are the $J, \mathrm{~L}, \mathrm{M}, \ldots$ absorptions associated with the emission of $J, L, M, \ldots$ electrons, and J, L, M, ... fluorescent (characteristic) radiations ${ }^{*}$.

The total mass absorption coefficient of $\mathrm{X}$-rays associated with fluorescence in any element may thus be written

$$
\frac{\tau}{\rho}=\left(\frac{\tau_{J}}{\rho}+\frac{\tau_{K}}{\rho}+\frac{\tau_{I}}{\rho}+\ldots\right),
$$

where $\tau_{K} / \rho$ is the absorption coefficient definitely associated with the emission of the $\mathrm{K}$ characteristic radiation, $\tau_{\mathrm{L}} / \rho$ is the absorption coefficient associated with the emission of $L$ radiation, and so on for each of the terms.

Each of the terms $\tau_{J}, \tau_{\mathrm{K}}, \tau_{\mathrm{L}}$, \&c. is zero when the wavelength of the primary radiation is greater than that of the constituent of shortest wave-length in the corresponding characteristic radiation; as the wave-length of the primary radiation becomes shorter, the term $\tau_{\mathrm{K}}$ or $\tau_{\mathrm{L}}$ suddenly rises and soon begins to diminish again with the wave-length, continuing to do so without limit. As all the $\mathrm{K}$ absorption curves are similar in form, $\tau_{\mathrm{K}} / \rho$ is a function of $\lambda / \lambda_{\mathrm{K}}$, thus for any element $\tau_{\mathrm{K}} / \rho=k f_{\mathrm{K}}\left(\lambda / \lambda_{\mathrm{K}}\right)$, the constant $k$ depending on the particular element. Similarly, $\tau_{\mathbf{L}} / \rho=l f_{\mathbf{L}}\left(\lambda / \lambda_{\mathrm{L}}\right)$. Presumably a similar relation may be found for $\tau_{\mathrm{J}} / \rho$.

The functions $f_{J}, f_{\mathrm{K}}, f_{\mathrm{L}}, \ldots$ though similar in features do not appear to be identical. Corresponding functions, however, are the same in different lements, and the relative values of the coefficients $j, k, l, \ldots$ appear to be the same for different elements. It should, however, be pointed out that the similarity of corresponding functions and equality of ratios of the corresponding coefficients have not been observed for substances differing widely in atomic weight. It may be found that these observed laws are not perfectly general. The particular form of these functions of $\lambda / \lambda_{\kappa}$ cannot be simply expressed; indeed, the exact form is not known in the regions where $\lambda$ is slightly less than $\lambda_{\mathrm{K}}$, owing to lack of perfect homogeneity in the radiations upon which experiments have been made. It has been shown, however, to be the same for each element, for the form has been found to be the same when instead of perfectly homogeneous radiations similar beams of radiations of neighbouring wavelengths have been employed.

* For more complete account see Bakerian Lecture 1916, Phil. Trans. A. 217. pp. 315-360. 


\section{Absorption Formulc.}

Various attempts have been made to express the absorption simply in terms of $\lambda$, but without mentioning these in particular it may be said that for the most part they give only rough averages over very limited regions; they are responsible for many inaccuracies.

As we have indicated,

Total absorption = scattering absorption + fluorescence absorption

$$
\begin{aligned}
\frac{\mu}{\rho} & =\frac{\sigma}{\rho}+\frac{\tau}{\rho} \\
& =\frac{\sigma}{\rho}+\left[\ldots \frac{\tau_{J}}{\rho}+\frac{\tau_{K}}{\rho}+\frac{\tau_{\mathrm{L}}}{\rho}+\ldots\right] .
\end{aligned}
$$

But $\sigma / \rho$ is a function both of the atomic weight $(\omega)$ of the absorbing substance and of the wave-length $(\lambda)$ of the radiation.

Thus

$$
\text { (3) } \frac{\mu}{\rho}=\mathrm{F}(\omega, \lambda)+\left[\ldots j f_{\mathrm{J}}\left(\lambda / \lambda_{\mathrm{J}}\right)+k f_{\mathrm{K}}\left(\lambda / \lambda_{\mathrm{K}}\right)+l f_{\mathrm{L}}\left(\lambda / \lambda_{\mathrm{L}}\right)+\ldots\right]
$$

As the relative values of the constants $j, k, l, \& c$. , seem to be the same for different substances, a single constant $n$ depending on the particular atomic weight or atomic number may be placed ontside the bracket, giving, within probable limits,

$$
\frac{\mu}{\rho}=\mathrm{F}(\omega, \lambda)+n\left[\ldots f_{\mathrm{J}}^{\prime}\left(\lambda / \lambda_{\mathrm{J}}\right)+f_{\mathrm{K}}{ }^{\prime}\left(\lambda / \lambda_{\mathrm{K}}\right)+f_{\mathrm{L}}{ }^{\prime}\left(\lambda / \lambda_{\mathrm{L}}\right)+\ldots\right]
$$

$\mathrm{F}(\omega, \lambda)$, however, varies little with the particular absorbing substance except when $\lambda$ is great. It is also approximately independent of $\lambda$ over a certain range of wave-lengths. The general form has already been indicated.

Consequently within certain limits equation (4) may be written simply

(5) $\frac{\mu}{\rho}=\mathrm{C}+k f(\lambda)$, where $k$ only depends on the particular

Hull and Rice, from the experiments on the penetrating radiations from a Coolidge tube, have recently pointed out that except when $\lambda$ of the absorbed radiation is near the

* This we have previously written in the form $\frac{(\mu / \rho-\mathrm{C})_{\mathrm{A}}}{(\mu / \rho-\mathrm{C})_{\mathrm{B}}}=k$, a constant for any two substances for any radiation of much more penetrating type than any characteristic radiation excited in either substance. 
wave-length of a characteristic radiation on the shorter wave-length side of the absorbing substance, $f(\lambda)$ becomes $\lambda^{3}$ simply. This is certainly very near to the truth when $\lambda$ is much less than $\lambda_{\mathrm{K}}$ the wave-length of the $K$ radiation of the absorbing substance. Aluminium is probably the best substance with which to test this relation, as the determinations of the absorption coefficient are probably more accurate than in any other substance. We have, therefore, plotted values of $\log (\mu / \rho-2)$ in aluminium against $\log \lambda$ over a long range of wave-lengths ( 39 to $2.28 \times 10^{-8} \mathrm{~cm}$.) greater than $\lambda_{J}$ for aluminium, using Barkla and Sadler's and our values for $\mu / \rho$ and Siegbahn's for $\lambda$. The relation is not exactly a linear one and shows that if we write $(\mu / \rho-2)=C \lambda^{n}, n$ is not a constant, but varies even over this range from about $2 \cdot 7$ to 3 . (No importance can be attached to the exact value $\cdot 2$ as it is usually very small in comparison with $\mu / \rho$, and is only introduced here as indicating a physical fact of which account must be taken over other ranges.)

We have shown, too, that the absorptions in other substances are proportional to those in aluminium for radiations of wave-length considerably shorter than those of the nearest spectral line. Thus, if it were true for aluminium it would be true approximately for other substances.

It should be remembered, however, that such a simple relation as is obtained by giving $n$ a constant value has not been found to hold accurately for wave-lengths anywhere really near to that of a $\mathrm{K}$ spectral line on its shorter wavelength side. In fact, if we can regard the absorption experiments as having given results approximating to those which would have been obtained with perfectly homogeneous radiations, the relation cannot hold oven to a first approximation when the wave-length is only slightly shorter than that of a $\mathrm{K}$ spectral line say. But further experiments are needed.

Hull and Rice, from their experiments on the absorptions in aluminium, copper, and lead, showed that $\mu / \rho-\cdot 12=k \lambda^{3}$ with a fair degree of accuracy and concluded that the scattering coefficient $(\sigma / \rho)=\cdot 12$.

It is probable, however, that a slight variation of $(\sigma / \rho)$ with $\lambda$ makes the scattering appear somewhat less than it actually is-at any rate with the longest waves used. It is highly improbable that the "fluorescence absorption" in this region is so strictly proportional to the cube of the wave-length as to enable us to use this as a method of determining $\sigma / \rho$ with any accuracy. 
The relation between $\mu / \rho$ and $\lambda^{3}$ is, however, so simple and convenient that we have plotted these two quantities for all the substances we have experimented upon.

The wave-lengths of short waves have been obtained from Hull and Rice's relation experimentally determined between wave-length and absorption in aluminium ; for longer waves Siegbahn's wave-lengths ( $\mathrm{K}_{a 1}$ lines) corresponding to Barkla and Sadler's and our absorption coefficients for the $K$ characteristic radiations have been used. The two sots overlap through only a very short range of wave-lengths, and this range is a particularly critical range, but the agreement is remarkably close and by the graphic method the results may be made perfectly continuous*. The wave-lengths obtained from these relations are given in Table I. columns 1 and 2 respectively. The two sets of wave-length determinations are distinguished by dots and circles in figure 2 , in which absorptions in copper, aluminium, water, and paraffin-wax are plotted against the (wave-length) ${ }^{3}$.

The discontinuities in the aluminium, water, and paraffin curves indicate again the positions of the $J$ spectral lines for aluminium, oxygen, and carbon. There is also a slight break in the copper curve suggesting a copper $\mathrm{J}$ radiation. But there may be some doubt as to this interpretation for the change is not so pronounced as we should have expected; it appears somewhat near to the $J$ lines for aluminium; and the fact that the two sets of values are confined to the two sides of the discontinuity leads us to treat the result with some caution. On the other hand, there are features in the relative absorptions in copper and other substances which suggest a change in the copper absorption in the neighbourhood of this particular wave-length (see aluminium absorption curve, figure 1); and we have no reason, beyond those stated, to question the accuracy of the result.

We thus see that over a limited range of wave-lengths the equation $\frac{\mu}{\rho}=\mathrm{C}+k \lambda^{3}$ (where $k$ is a constant for a given substance) fairly accurately represents the experimentally observed relation-very much more accurately indeed than any simple tormula of the type $\mu / \rho=k \lambda^{n}$. It, however, does not express the fact of the variation of $\sigma$ with $\lambda$ such as has been described, nor does $k \lambda^{3}$ appear to hold at all accurately

* It is unfortunate that Hull and Rice continued their experiments only up to the point where the discontinuity occurred both in aluminium and in copper. 
except for wave-lengths far removed from those of the characteristic radiations excited in the absorbing substance.

Fig. 2.

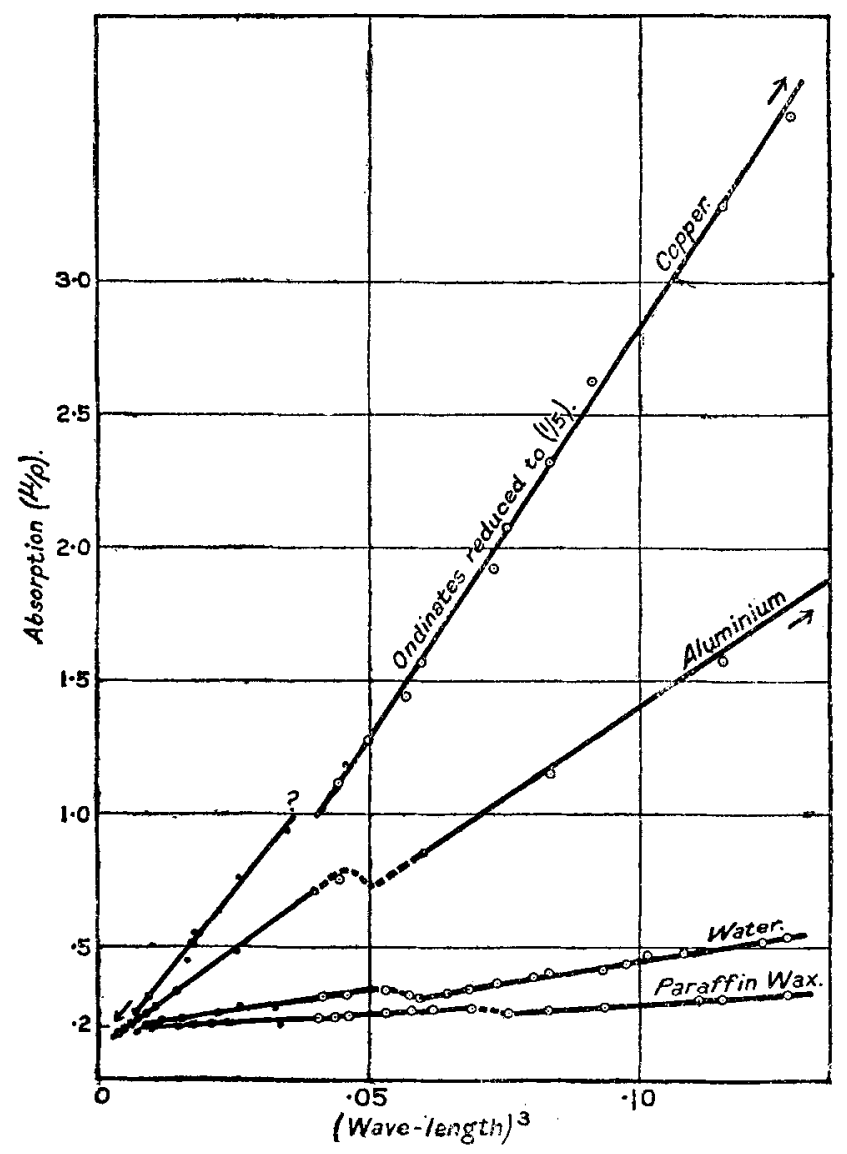

There were one or two minor features in our experiments which we may just mention, though we have not yet had time to investigate them fully. In the absorption curve for paraffin, for instance, there appeared to be a consistent slight irregularity at certain wave-lengths. When the absorption $(\mu / \rho)$ in copper was about $3 \cdot 5$, or $\lambda=\cdot 29 \times 10^{-8} \mathrm{~cm}$., certain rather high values were obtained for the absorption in paraffin, values say 10 per cent. above the normal. There seems no doubt that these irregularities really exist, but a permanent increase in the absorption for wave-lengths 
shorter than a certain value has not been observed. This, however, can be accounted for if corresponding radiations in other elements are of neighbouring wave-length.

Similar irregularities were found in the absorptions by paper and water, both within the same region.

It seems just possible that these are connected with a spectral line or spectral lines of still higher frequencypossibly of an I series. This, however, is a point which would require careful investigation by another method.

It should also be pointed out that though we have drawn smooth curves-for the most part straight lines-through observed points, this does not indicate that certain slight deviations are not real. We know certain of them to be so. but they are only small, as the figures show quite clearly. For the present they are of only secondary importance.

We wish to acknowledge our indebtedness to Miss $J$, Dunlop for some preliminary work on the absorption of high-frequency X-rays.

XXX. The Limiting Frequency in the Spectra of Helium, Hydrogen, and Mercury in the Extreme Ultra-Violet. By O. W. Richardson, F.R.S., Wheatstone Professor of Physics in the University of London, and Lieut. C. B. Bazzoni, General Staff A.E.F., formerly Harrison Research Fellow of the University of Pennsylvania*.

$r$ HE investigations described in this paper were undertaken for the purpose of detecting and measuring the frequency of the shortest vibrations emitted from the various gas atoms under electron impacts. A preliminary notice of some of the results obtained has already been published in 'Nature' $\dagger$. From the quantum relation $e \mathrm{~V}=h \nu$ a certain frequency $\nu$ can be calculated for each gas from its ionization potential $\mathrm{V}$ which should be the maximum frequency obtainable from that particular gas when subject to the same type of ionization. This calculation gives a probable minimum wave-length for hydrogen of $909 \times 10^{-8} \mathrm{~cm}$. and for helium of $422 \times 10^{-8} \mathrm{~cm}$. assuming Bohr's ionization potentials $(13 \cdot 6$ volts for hydrogen and $29 \cdot 3$ volts for belium) to be correct. In making these computations the value of $e$ is taken to be $4.77 \times 10^{-10} \mathrm{E} . \mathrm{S}$. unit and of $h 6.55 \times 10^{-27}$. Taking the experimentally determined values of $10^{\circ} 4$ volts

* Communicated by the Authors.

+ Richardson and Bazzoni, 'Nature,' vol. xcviii. p. 5 (1916).

Phil. Mag. S. 6. Vol. 34. No. 202. Oct. 1917. 